\title{
Enhancing Student Learning and Engagement in the Course on Computer Networks
}

\author{
Jeyamala Chandrasekaran ${ }^{1}$, Anitha $\mathrm{D}^{2}$, Thiruchadai Pandeeswari $\mathrm{S}^{3}$ \\ ${ }^{1}$ Department of Information Technology, Thiagarajar College of Engineering, Madurai, Tamil Nadu, India \\ ${ }^{2}$ Department of Applied Mathematics and Computational Science, Thiagarajar College of Engineering, Madurai, Tamil Nadu, \\ India
}

${ }^{3}$ Department of Information Technology, Thiagarajar College of Engineering, Madurai, Tamil Nadu, India

\section{1jeyamala@tce.edu \\ ${ }^{2}$ anithad@tce.edu \\ 3 eshwarimsp@tce.edu}

Abstract: The course on Computer Networks plays a significant role in the professional development of students graduating from various programmes such as Computer Science and Engineering, Information Technology and Master of Computer Applications. Graduating Engineers with exclusive skills in administering and managing computer networks are highly demanded in the job market. The knowledge on computer networks is tested in many of the entrance examinations towards admission in Post Graduate programmes like Graduate Aptitude Test in Engineering (GATE), Tamil Nadu Common Entrance Examination (TANCET) etc., The course also provides the necessary foundation for certification exams like Cisco Certified Networking Associate (CCNA). Having realized the importance of the Computer Networks in placements, higher studies and international certifications, the instructional design has been evolved with the appropriate selection of active learning and collaborative learning strategies. The paper presents a detailed impact analysis of the instructional design in improving the attainment of learning outcomes, student engagement and sarisfaction. The experimental study has been carried out in a student group of 130 members from the undergraduate programme on Information Technology at Thiagarajar College of Engineering, Madurai in the academic year 2020-21. A wide variety of network simulation tools, programming assignments have been included in the instructional design to promote hands on experience. Exclusive assessment items with customized rubrics are used for promoting technical, personal and interpersonal skills. The blend of instructor led and inquiry based teaching learning practices have shown significant improvement in the grades of continuous assessment test

\footnotetext{
Corresponding Author

Jeyamala. C

Department of Information Technology,

Thiagrajar College of Engineering, Madurai.

jeyamala@tce.edu

and terminal examinations. A positive impact in promoting engagement of learners has been realized from the course end surveys. The capability for self-learning has been measured from the performance in online certification courses which were beyond the curriculum.
}

Keywords: Computer Networks, Active Learning, Collaborative Learning, Learning Outcome Attainment, Higher Order Thinking Skills, Student Engagement

\section{Introduction}

The course on Computer Networks is offered to students of various engineering disciplines during their second year of study. When they reach their fourth semester to learn this course on Computer Networks, they would have finished learning courses that deal with

- Basic networking and web development

- Programming and application development using C, Python and Java frameworks

- Tools and techniques required for application provisioning over internet.

- Therefore the only piece missing from the puzzle of understanding novel computing systems is 'Networking'. Hence, a course on computer networks that gives students a complete understanding, ability to design and work with the network backbone between Clients and servers becomes essential. Considering these requirements, the computer networks course has been designed with the objective of providing indepth knowledge on various protocols and mechanisms required to establish communication in the global internet scenario.

Though the course has been designed to provide enriched exposure to a whole set of algorithms, mechanisms and tools required as part of the data communication networks, there are some major challenges in delivering the content effectively. One of the challenges observed is that students tend to learn these concepts in an abstract manner with no to very limited perspective of adapting networking requirements to that of application. The following are some of the sample scenarios that highlight the need of learning the networking concepts in-depth and at higher cognitive levels

Scenario 1 - Student may develop a novel distributed application that requires specific handshaking algorithm 
to be implemented to ensure the live status of the involved nodes

Scenario 2 - Student may develop an IoT application that require a specific machine to machine communication protocol to be adapted at transport layer

Scenario 3 - Student may develop a project that makes use of bio inspired techniques to solve routing problems

Many such scenarios may be listed. In order to handle the above scenarios successfully, it is essential that the students imbibe the concepts of networking at higher cognitive levels and abstract learning will be of very little use.

Second major challenge observed is that such a content delivery plan was not helping to a greater degree in promoting the higher order thinking skills among the students. Though students learn these algorithms, a customized instructional design that helps the students to internalize the concepts is required. An Instructional design that promotes inquiry based learning, collaborative activities, student engagement at higher cognitive levels is required. It is paramount to involve rich set of simulation tools, novel collaborative exercises, activities that promote self-learning in the instructional design.

\section{Related Works}

Significant amount of research has been carried out on the pedagogy for computer networks. Zhang et al. have carried out an experimental investigation on the use of Packet Tracer, a visualization tool in enhancing the creativity and innovation of students. Positive feedbacks from students and teaching team have confirmed the effectiveness of the adopted teaching practice. One of the important problems highlighted is that the students will not be able to access real networking equipment like hubs, switches and routers as and when they want. And the other problem highlighted is that the students not being able to visualize the data packets going in and coming out of the devices. These problems have been addressed by assigning mini-projects based on different problem statements to the students.

Effectiveness of the use of Packet analyzer tool in teaching learning has been evaluated (Yang, 2019). The study evaluates the efficiency of learning computer networks course with cisco learning platform and packet tracer tool. The effectiveness of the content delivery plan is evaluated through students' feedback obtained on a questionnaire and grades obtained by students.

Number of problems such as multifaceted nature of the course that involves learning basic network principles, working of network equipments and network design, failure of classroom-oriented teaching in arousing students curiosity and creativity, insufficient network equipments leaving no opportunity for hands on practice are highlighted. (Zhang, 2016). A new teaching model (SPOC - small private online course) has been proposed to overcome the above-mentioned problems. The experimental results show that the new teaching model has enhanced the learning potential of the students to a greater degree.

Flipped classrooms aligned with learner centric teaching learning methods have been leveraged to improve the quality of content delivery in computer networks course.(Knezvic, 2019). Flipped classroom model proposed in comprises of practical activities, Q\&A sessions, group activities and quizzes as in-class activities. Learning materials from cisco learning academy and packet tracer tool were made use of in the content delivery.

Practical exercises are considered to be very crucial for enhancing the learning of computer networks. Need for hands on configuration experiences, experiments that enhance students to review basic theory concepts, heuristic based experiments that enhance critical thinking of students, simulation-based experiments that enable students to explore modern networking technologies are emphasized. (Gao, 2018) The conduct of the lab sessions is accompanied by number of Q\&A sessions and discussion forums online.

Flipped classroom mode for the practical exercises has been experimented.(Guan, 2017) The flipped classroom based methodology proposed includes both in-lab and out-of-lab teaching activities. The teaching activities are carried out both online and offline. The online lab resources usually include network simulation software, virtual desktop that allows students to login remotely. The offline lab resources usually include networking equipment such as routers and switches. The experiments are backed up by a detailed experimental plan and number of online Q\&A sessions and discussion forums.

Use of the virtualization tool Netkit has been investigated . (Gurgel, 2013). In this paper, a virtualization tool based on a novel approach called Integrated Modelling approach has been proposed. The content delivery plan proposed makes use of the Netkit virtual tool on top of specially designed educational modules. The modules contain materials designed based on three models viz conceptual, Instructional and didactic. The conceptual model comprises of description of the domain under study. The didactic model comprises of sequence of important elements generated in other two models. This ensures reinforcement learning. Use of such tool would be more suitable in distance learning set up where the student does not have infrastructure to install and configure a wide set of tools to learn different networking concepts.

From the literature survey carried out, the following issues were identified pertaining to teaching computer networks course :

- The computer networks course is inherently multifaceted as it requires learners to learn basic concepts, logical sequence of actions, working principle of networking devices, management of 
networking devices, design of algorithms and implementation of algorithms

- Lack of visualization of data processing at nodes and data transmission through them.

- Extensive and expensive lab set up to facilitate effective conduct of experiments

- Practical exercises that more often require students to follow the detailed manual provided by the instructor step-by-step which does not require in-depth thinking.

- Less scope for hands on experience on real networking devices

- Less scope for exploring domain related problems offline/out-of-lab

- Networking technologies are evolving faster especially after the advent of IoT

The above mentioned issues have been addressed in number of studies made on evolving teaching learning methods in particular for the computer networks course. However, a customized Instructional model that focus on blend of instructor led and inquiry based approaches aligned with the use of simulation tools and rubric based open ended assessments especially for teaching computer networks is still an uninvaded arena.

\section{Research Question}

The motivation for research is supported by the following research questions:

A. What is the impact of instructional design with active learning and collaborative strategies in enhancing the attainment of learning outcomes in the course on computer networks?

B. What is the impact of inquiry based teaching with simulation tools in promoting student engagement and satisfaction?

\section{Materials and Methods}

\section{A. Course Details}

The course on Computer Networks enables the students not only to strengthen their knowledge on Network models, technologies and protocols, but also promotes skill development in socket programming, network simulation and packet analysis. The detailed contents of the course and the assessment pattern are provided in the link https://tinyurl.com/TCECN2018. The course outcomes listed in Table1 are mapped in accordance with bloom's taxonomy in cognitive, psychomotor and affective domain. Canvas has been used as a learning management system for both the theory and practical courses.

\section{Table 1. Course Outcomes}

$\begin{array}{lll}\text { CO } & \text { Course Outcomes } & \begin{array}{l}\text { Blooms } \\ \text { Level }\end{array} \\ \text { CO1 } & \begin{array}{l}\text { Describe various Network components, } \\ \text { topologies, reference models and } \\ \text { technologies }\end{array} & \begin{array}{l}\text { Understand } \\ \text { EO2 }\end{array} \\ & \begin{array}{l}\text { Experiment Flow control, Error control and } \\ \text { Access control techniques at Data link layer }\end{array} & \text { Apply } \\ & \end{array}$

CO3 Apply Subnetting and routing mechanisms for a given network

CO4 Explain the working principle of End-toEnd and application layer protocols

CO5 Compare the needs and implementation architectures of peer to peer, Client server and Cloud Networks

CO6 Examine the Performance metrics of a specific Network traffic using tools such as Wire shark

B. Instructional Design with Socket Programming, Network Simulations and Animations

In order to enhance the learning outcomes, a detailed course plan that involves the usage of simulations, animations and network analysis is developed. The instructional design includes a rich blend of hands on experiments exclusively on socket programming, network simulation and protocol analysis. Socket Programming exercises were carried out using networking APIs in Java. Simulation Experiments were carried out using GNS3 or Riverbed Modeller Academic edition. Packet Analysis experiments were carried out using Wireshark, Fiddler or Network Miner. Wireshark is a networking tool widely used for packet analysis. In the module on transport and network layer, wireshark has been used for conducting experiments related to the analysis of various fields present in TCP, UDP and IP protocols. The activity posed by the instructor on using wireshark for IP datagram frame format is depicted in Figure 1. An instance of submission made by the students is depicted in figure 2

Students also had an experiential learning on the usage of OPNET for simulation of routing protocols like Distance Vector Routing, Link State Routing, Open Shortest Path First (OSPF) and various networking topologies and its corresponding performance analysis. An instance of student submissions on simulations of RIP has been presented in Figure 3.

Every module in the course is covered with appropriate animations to promote student engagement and learning. Figure 4 highlights the usage of network animations used in the course.

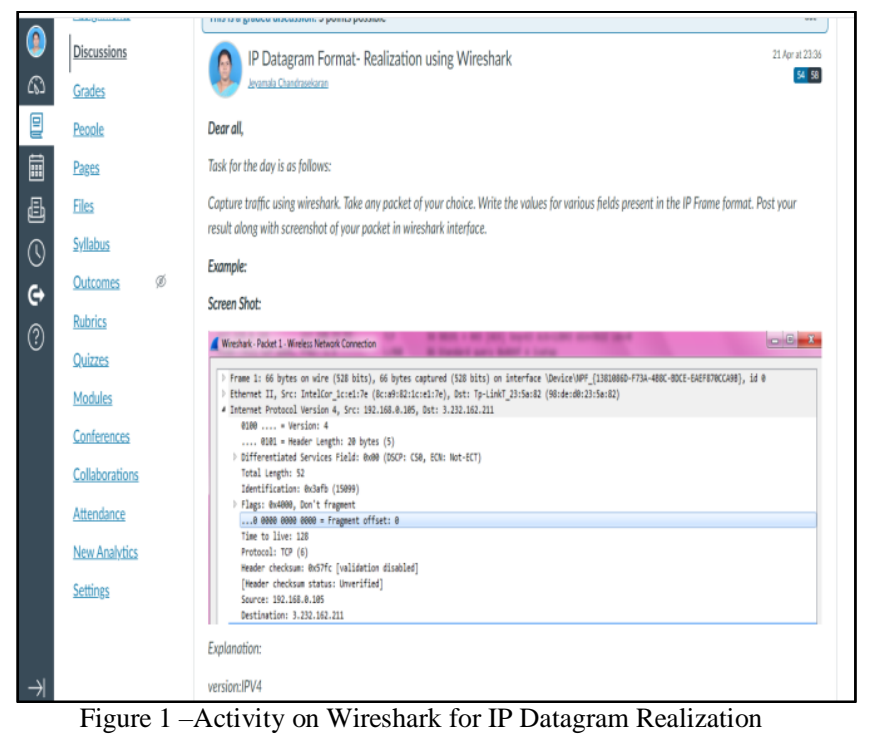




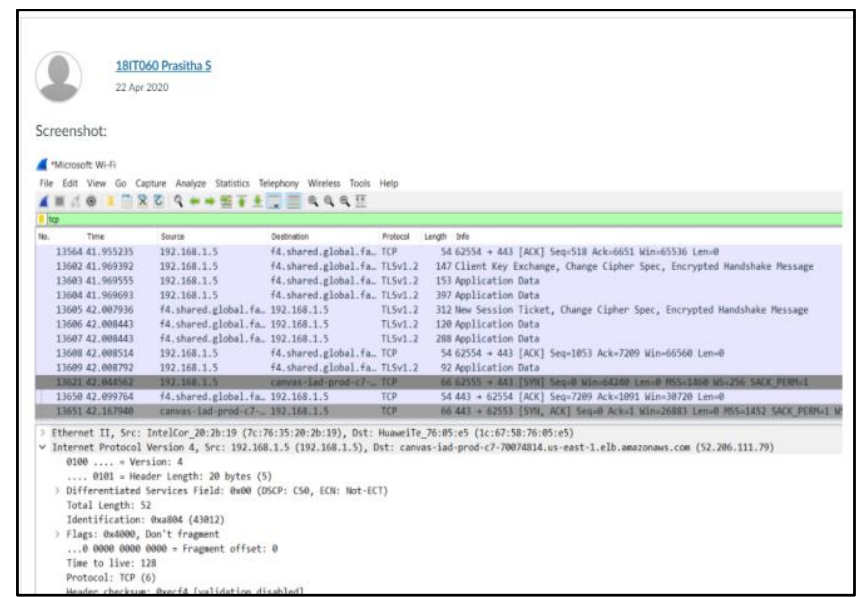

Figure 2-Student submissions on using Wireshark for IP datagram realization

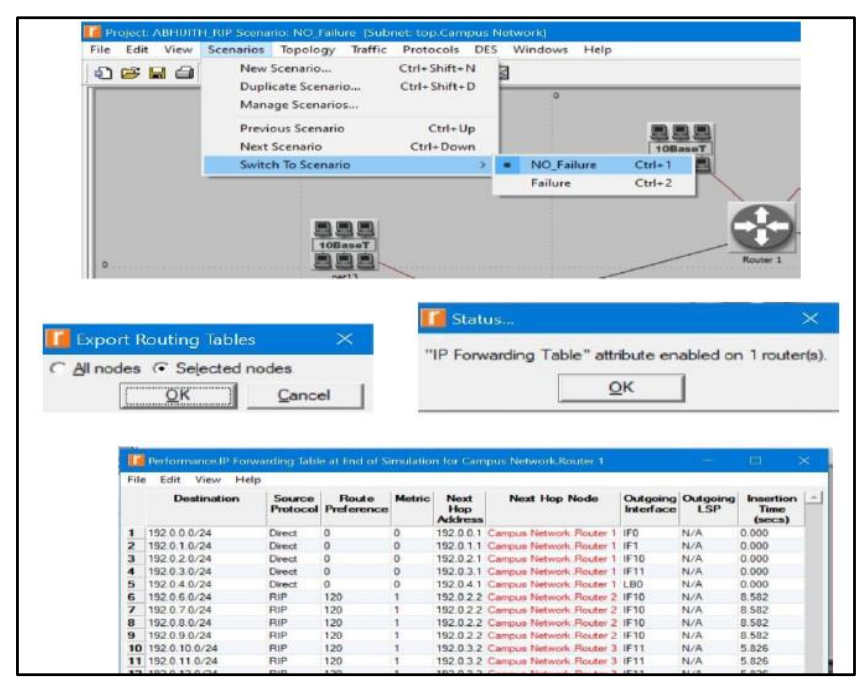

Figure 3 -Simulation of RIP using OPNET

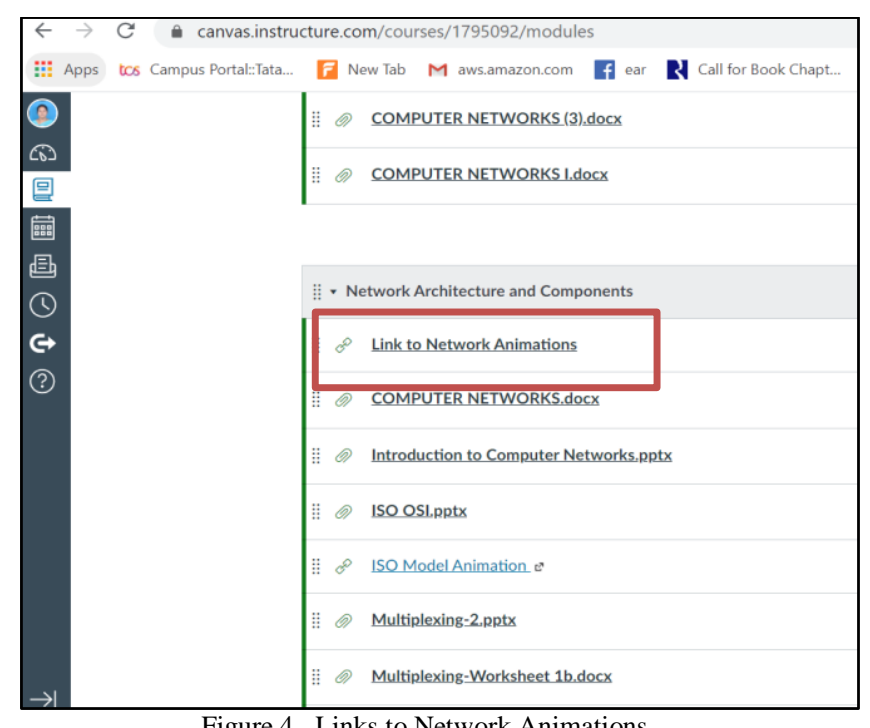

Figure 4-Links to Network Animations
C. Design of Worksheets to Promote Problem Solving Skills

Exclusive worksheets were designed for every module with problems focussing on higher order thinking skills like "Apply" and "Analyze". These worksheets helped the students to work with a variety of problems corresponding to different networking scenario. Few problem statements were taken from GATE question banks and CCNA certification exams. Solving worksheets as a part of assignment has resulted in significant improvement in the performance in Continuous Assessment Tests and in Terminal Examinations. A sample worksheet used for Distance Vector Routing is presented in Figure 5.

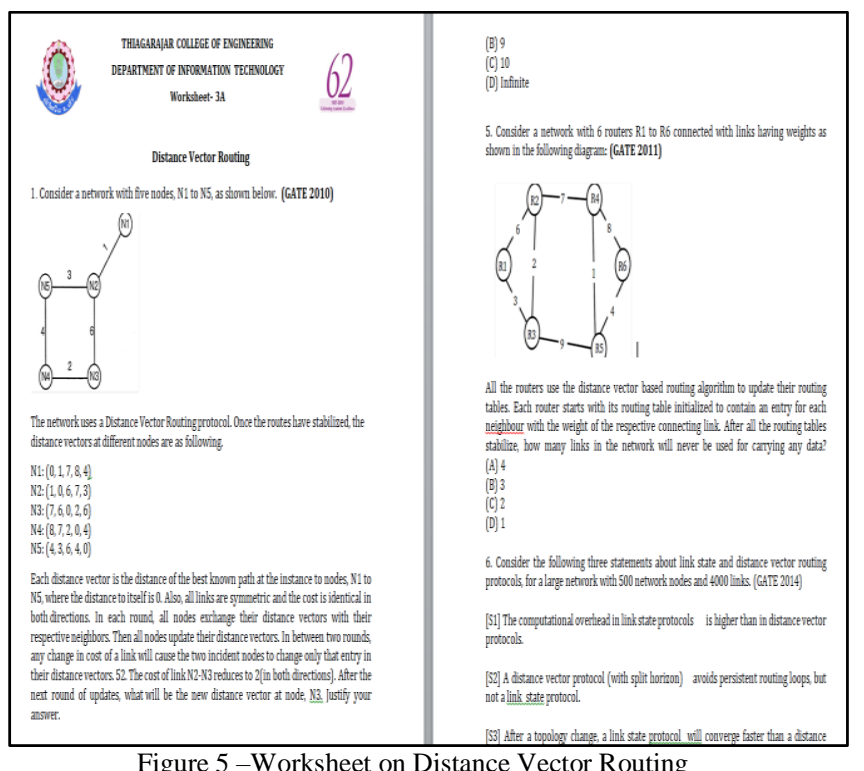

Figure 6 presents a screenshot of worksheets on different topics which were shared in canvas.

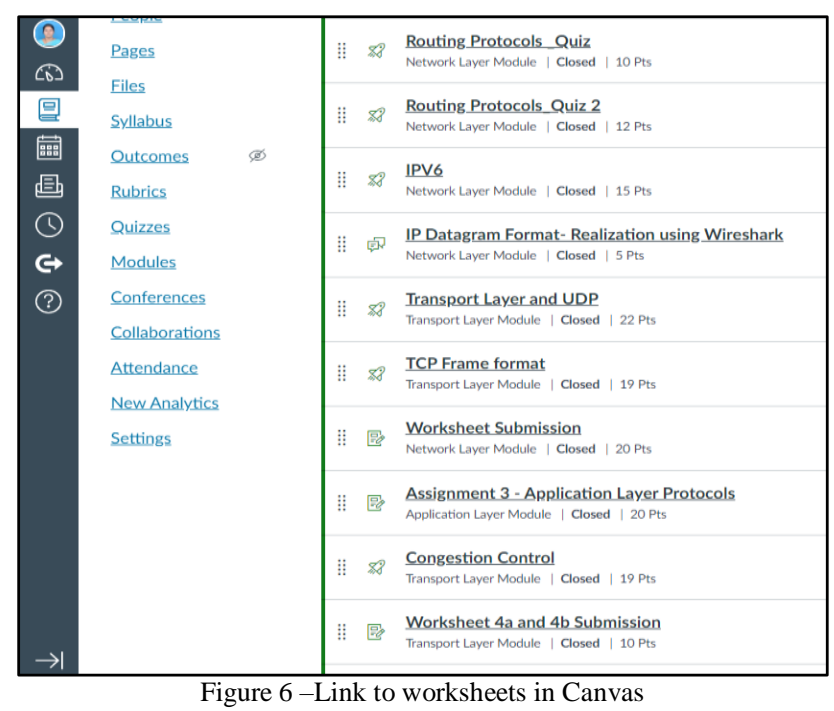

D. Field Visits 
In order to provide first-hand experience on local area networking and to promote inquiry based learning, this activity was formulated. As part of this activity, students would form teams and as teams they were asked to visit the various labs in the departments and observe the networking cables and equipment present in the lab. Almost all the labs have D-Link layer 2 switches with varying number of ports. One of the labs also has a rack of routers. Students were asked to observe the networking structure, equipment and prepare a one page write-up on the LAN present in their department. They were also made to visit the datacentre present in our college, where they observed whole lot of networking switches and other equipment. This field visit was designed as a guided tour, where in the technicians present in the labs and datacentre helped the students to get better understanding on the networking infrastructure present in the college premises.

\section{E. Learning by Doing}

This activity was formulated to improve their understanding of various mechanisms and related algorithms used for implementing these mechanisms at data link layer and network layer. Learners were asked to choose one of the many mechanisms they learnt and algorithm to implement the same. Students were asked to prepare creative models ranging from videos, animations, story boards, demo boards, illustrative chart works etc., to explain their understanding the chosen mechanism and the algorithm. Peer grading has been enabled for these assignments. The students are also expected to program various algorithms in data link and network layers. They were also asked to find out a suitable real life problem analogy for the purpose served by the mechanism under study. This activity helped them to look at the mechanisms and algorithms at various contexts and give them an opportunity to learn these algorithms in a meaningful manner. Each creative work carried out was presented to the entire class. Figure 7 and 8 represent an instance of programming assignments and animations and Table 2 indicates the rubrics used for peer assessment of the same.

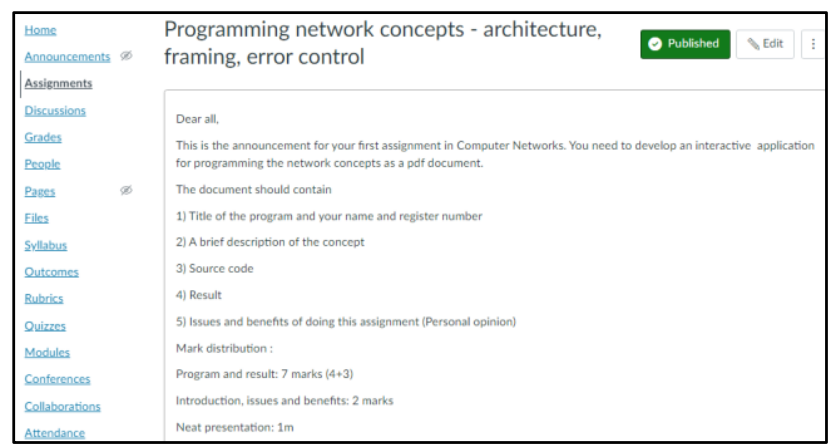

Figure 7 Programming Assignment

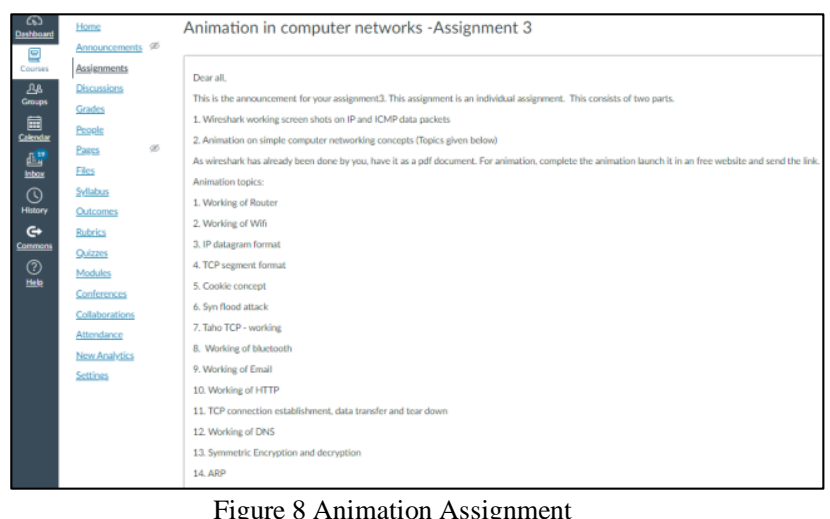

Table 2. Peer grading Rubric for animation assignment

Parameters

Points

Quality of Content (Quantum and correctness of content )

Creativity, Interesting to watch

2.0

Modern tool usage

1.0

Userfriendliness and interactiveness

1.0

\section{F. Online Quizzes for Formative Assessment}

Online quizzes have been effectively utilized to promote reinforcement of learning. Quizzes have been created for every module with questions addressing lower order thinking skills and also higher order thinking skills. Online quizzes have also served as an effective tool for self-assessment. Learners were able to understand the expected depth of learning required for the course. Figure 8 reports the various quizzes designed for conducting formative assessments.

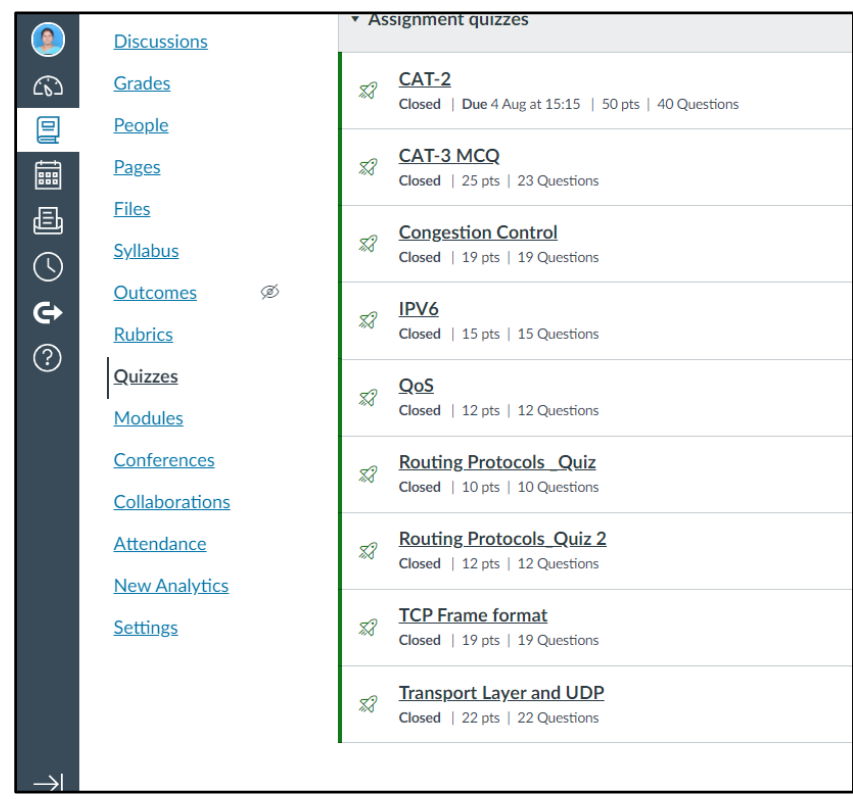

Figure 8 - Online Quizzes for Formative Assessment 
G. Design of Assessment Items to Promote Hands On Experience and Team Work

Creating, managing, securing, and administering computer networks in reality involve a wide usage of supporting tools and techniques. An exclusive team assignment was created for the learners to explore any network management / security tool of their choice. The procedure for installation and illustration of functionalities are to be included in the report. Learners were made to develop a worksheet which contains simple hands on exercise and problems to test the expertise in the corresponding tool usage. The presentation reports and the worksheets served as an excellent resource for peers who are interested in learning different tools. The reports have been graded using exclusive parameters as reported in the Table 3. Learners were exposed to 15 different tools like PRTG Monitor, The Dude, OpenSSL, NMAP, Fiddler etc., The assignment has also created an opportunity for learning from peers.

\section{Table 3. Grading Parameters for Assignment}

\section{Parameters}

Selection of Appropriate tool

Clarity in Installation procedure

Clarity in Capability Demonstration

Quality of worksheet

Contribution of Individual

Quality of Presentation

Total

Grade Points
5
5
5
5
5
5
30

H. Design of Assessment Items to Promote Self -learning and communication

To promote self-learning and communication, learners were made to create video presentations to illustrate the working principle of application layer protocols like HTTP, SMTP, HTTPS, TELNET, FTP etc., Rubrics have been shared amongst the students and is presented in Figure 9.

\begin{tabular}{|c|c|c|c|}
\hline Parameters & Excellent & Good & Fair \\
\hline $\begin{array}{l}\text { Depth of the } \\
\text { content }\end{array}$ & $\begin{array}{l}\text { Basic and } \\
\text { advanced } \\
\text { functionalitie }\end{array}$ & $\begin{array}{l}\text { Little basic } \\
\text { functionality } \\
\text { is explored. }\end{array}$ & $\begin{array}{l}\text { Limited } \\
\text { functionalitie } \\
s \text { have been }\end{array}$ \\
\hline & $\begin{array}{l}\mathrm{s} \text { of the } \\
\text { protocol are } \\
\text { explored in } \\
\text { detail with } \\
\text { examples }\end{array}$ & $\begin{array}{l}\text { The depth of } \\
\text { coverage is } \\
\text { moderate }\end{array}$ & $\begin{array}{l}\text { explored. No } \\
\text { illustrations } \\
\text { or examples } \\
\text { have been } \\
\text { provided }\end{array}$ \\
\hline $\begin{array}{l}\text { Organization of } \\
\text { the content }\end{array}$ & $\begin{array}{l}\text { The contents } \\
\text { are well } \\
\text { organized and } \\
\text { presented in a } \\
\text { lucid manner }\end{array}$ & $\begin{array}{l}\text { The contents } \\
\text { are not } \\
\text { organized in a } \\
\text { coherent } \\
\text { manner }\end{array}$ & $\begin{array}{l}\text { Improper } \\
\text { organization } \\
\text { of content. } \\
\text { No adherence } \\
\text { to the } \\
\text { templates } \\
\text { provided }\end{array}$ \\
\hline $\begin{array}{l}\text { Communicatio } \\
\mathrm{n}\end{array}$ & $\begin{array}{l}\text { Language } \\
\text { used is } \\
\text { excellent and } \\
\text { no } \\
\text { grammatical } \\
\text { errors }\end{array}$ & $\begin{array}{l}\text { Language } \\
\text { used is } \\
\text { understandabl } \\
\text { e with fewer } \\
\text { grammatical } \\
\text { errors }\end{array}$ & $\begin{array}{l}\text { A lot of } \\
\text { grammatical } \\
\text { errors and } \\
\text { spelling } \\
\text { mistakes }\end{array}$ \\
\hline Citations & $\begin{array}{l}\text { All references } \\
\text { have been }\end{array}$ & $\begin{array}{l}\text { Only a few } \\
\text { references }\end{array}$ & $\begin{array}{l}\text { No citation } \\
\text { for references }\end{array}$ \\
\hline
\end{tabular}

\begin{tabular}{|c|c|c|c|}
\hline & cited & $\begin{array}{l}\text { have been } \\
\text { cited }\end{array}$ & \\
\hline $\begin{array}{l}\text { Timely } \\
\text { Submission }\end{array}$ & $\begin{array}{l}\text { Submitted on } \\
\text { Time }\end{array}$ & $\begin{array}{l}\text { Submitted two } \\
\text { days late }\end{array}$ & $\begin{array}{l}\text { Late } \\
\text { submission } \\
\text { by more than } \\
\text { three days }\end{array}$ \\
\hline
\end{tabular}

I. Content beyond Curriculum - Integration with NPTEL course

Learners were made to register in the NPTEL course on Computer Networks and Internet Protocol and were mentored by the instructors for completion of weekly assignments. Assignment Scores in the NPTEL course were counted for continuous assessment. The details of the mentee in NPTEL are presented in Figure 8.

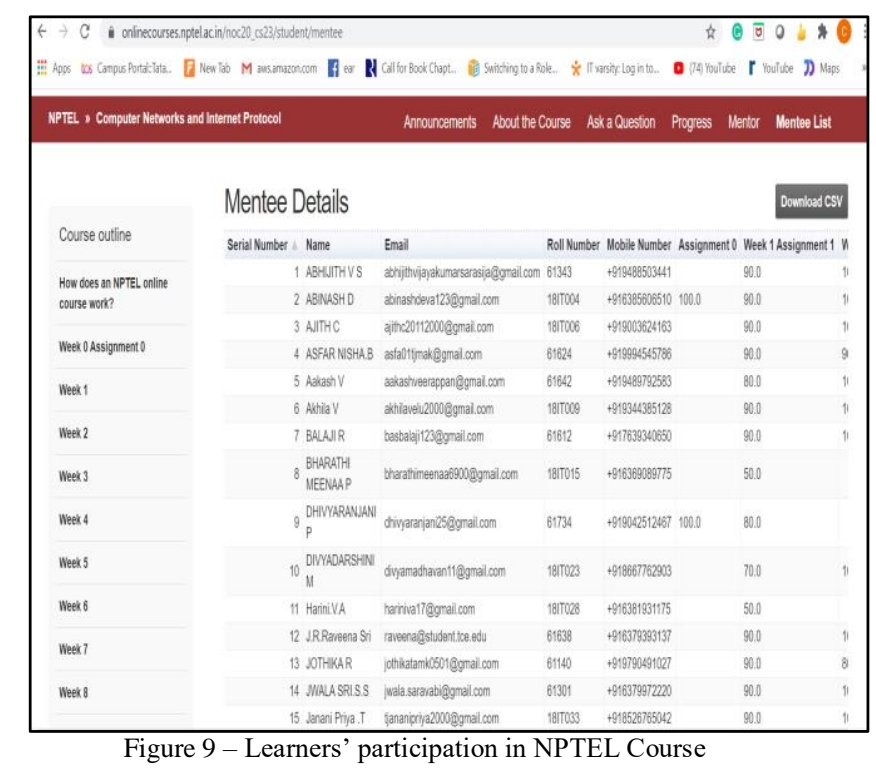

\section{Experimental Results}

A. Impact on the Attainment of Learning Outcomes.

In support of Research question 1, the performance of the students in Continuous Assessment Test and Terminal examinations are analysed and are presented in Figures 10 and 11 respectively.

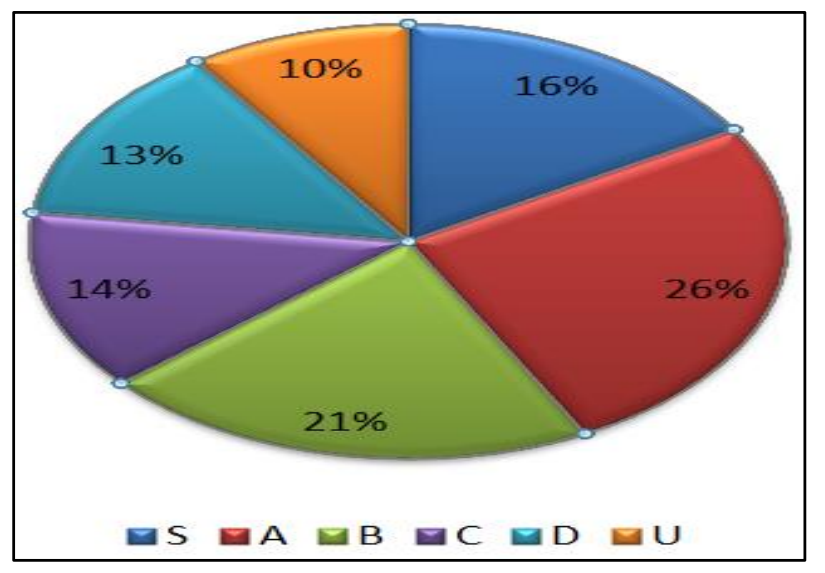

Figure 10 - Students' Performance in Continuous Assessment Tests 
It could be inferred from the Figure 10 that the count of failures in terminal examinations for the experimental group has significantly reduced to $2 \%$. From figures 10 and 11 , it is evident the count of failures in terminal examinations is relatively low when compared with the students performance in continuous Assessment Tests. The performace of the students has also been compared with the performance of students during the previous four academic years and the results have been presented in the figure 12. The count of failures has reduced drastically and is the lowest, when compared with the results of previous batches. More than $68 \%$ of the students in experimental group has scored $70 \%$ and above. The improvement in grades clearly demonstrates the effectiveness of the adopted instructional design in enhancing the attainment of learning outcomes.

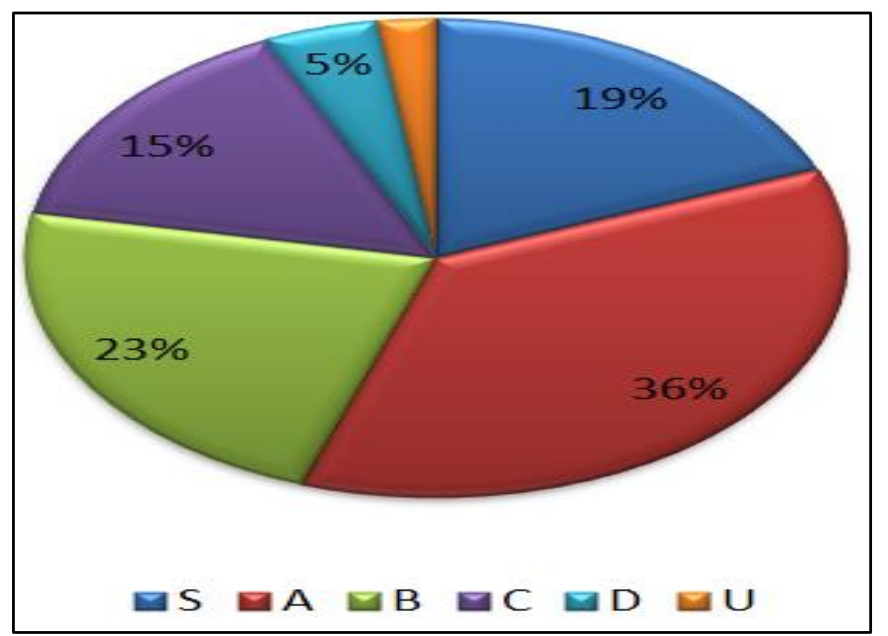

Figure 11 - Students' Performance in Terminal Examinations

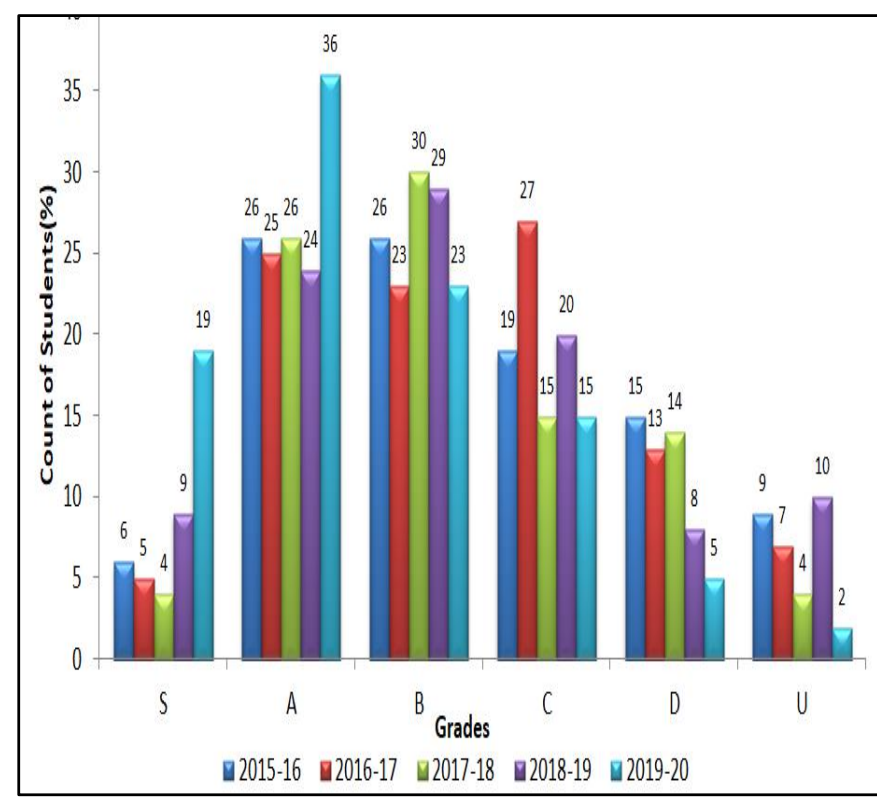

Figure 12 - Performance comparison of experimental group with previous batches
The activity on field visits as discussed in the previous section has created a lot of curiosity among students. Learners came up with many questions and these questions were set as their objectives of learning. This improved student engagement to a greater extent and also increased their self-learning quotient by manifolds. Figure 13 shows the comparison of students performance in the internal exams especially on the questions relevant to Local Area Networking technologies. It can be observed from the graph that, the batch that was given the field visit activity performed better than the controlled group which didn't carry out the activity.
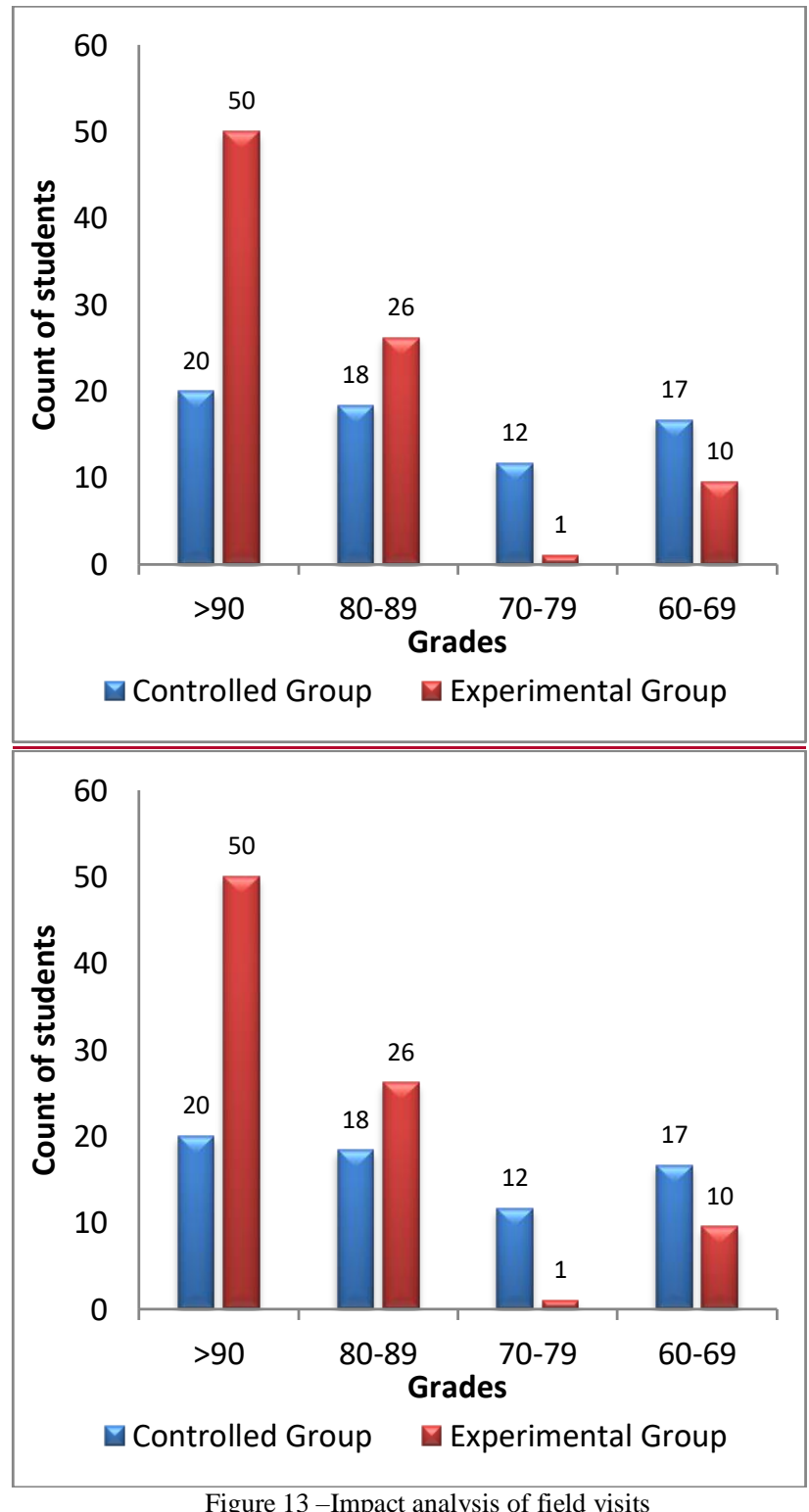

The activity on making videos, model, simulations and prototypes have helped the learners to look at the mechanisms and algorithms at various contexts and give them an opportunity to learn these algorithms in a meaningful manner. Each creative work carried out was presented to the entire class. This paved 
way for peer learning. Impact of this activity can be studied by comparing the performance of the batch of students that underwent this activity and the batch that didn't. The comparison chart is presented in Figure 14.
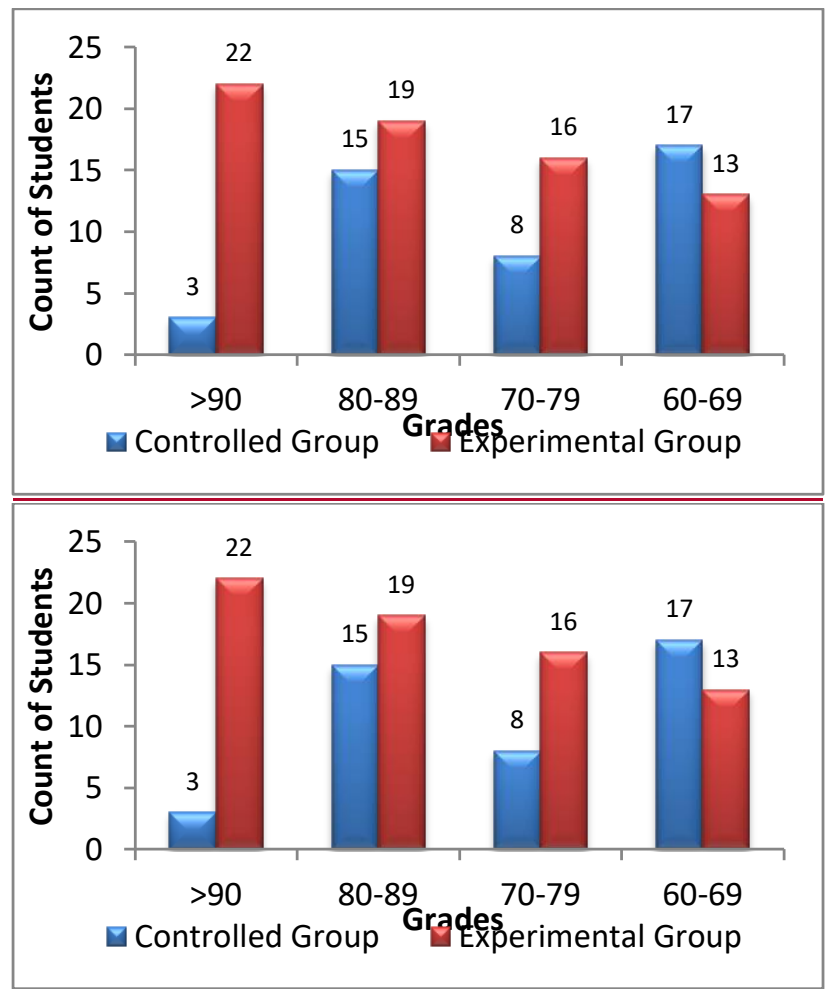

Figure 14-Impact analysis of learning by doing

A course exit survey was also conducted on a likert scale of 1 to 4 on various parameters related to course content, attainment of course outcomes, content delivery and assessment. The score for each parameter is calculated by considering the count of top two ratings in the total number of responses received. It could be inferred from the figures 15 and 16, that the overall scores for the effectiveness of course content is $78.6 \%$ and attainment of course outcomes is $76.84 \%$ effectively. The reasonable satisfaction index confirms the effectiveness of the instructional design which has a right blend of active and collaborative learning strategies.

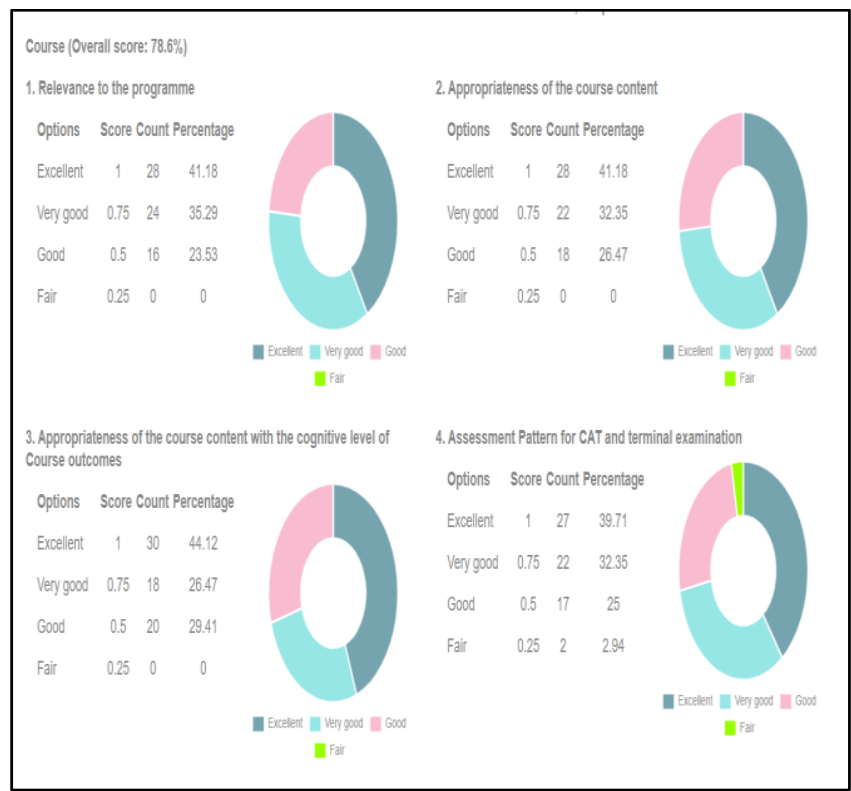

Figure 15 - Course Exit Survey - Analysis of parameters on Course Content

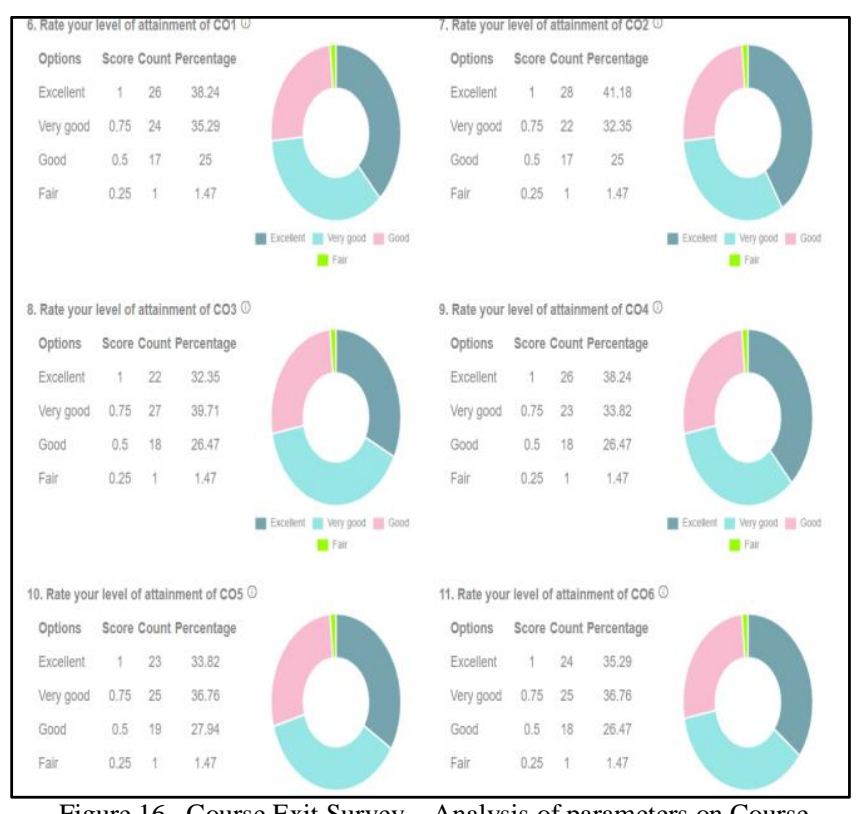

Figure 16- Course Exit Survey - Analysis of parameters on Course Outcome Attainment

B. Impact on Promoting Student Engagement and Satisfaction

For support of Research Question 2 on the impact of instructional design towards student engagement and satisfaction, parameters on the content delivery and assessment in course exit survey, qualitative feedbacks, participation in related online courses on Computer Networks and Internet protocol in NPTEL and Open University are considered as evidences. It could be inferred from figure 17 that, the overall score for content delivery is $79.8 \%$. Figure 18 indicates that $99.8 \%$ of learners felt they were actively engaged in the classes. Around $95 \%$ of the students supported the usage of active 
learning strategies like group discussions, role plays, flipped classrooms etc., which were used in the classes.

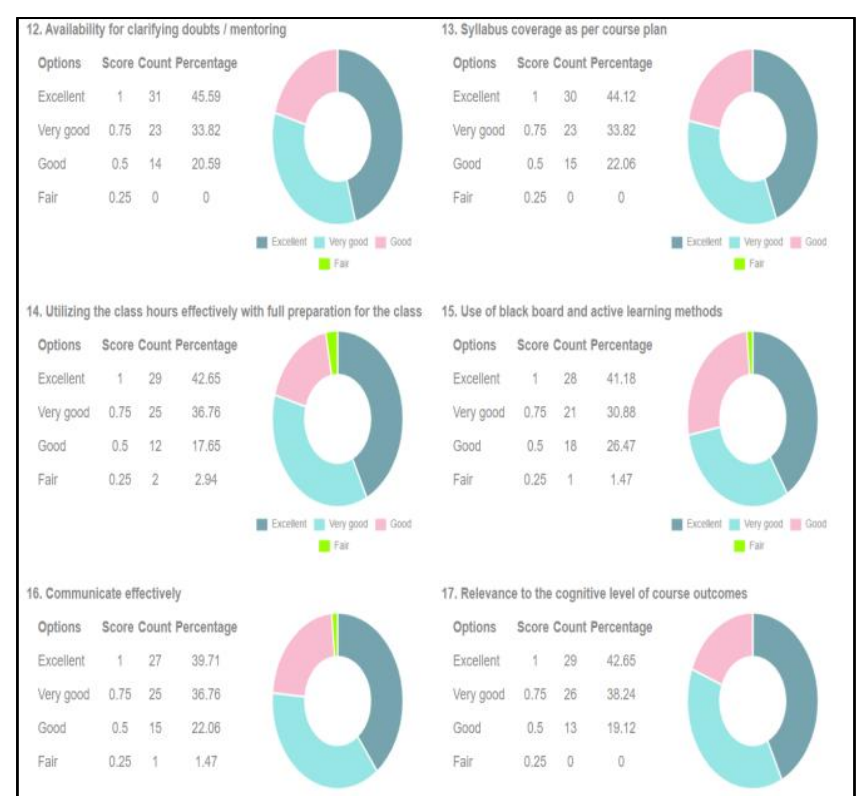

Figure 17- Course Exit Survey - Analysis of parameters about Content Delivery

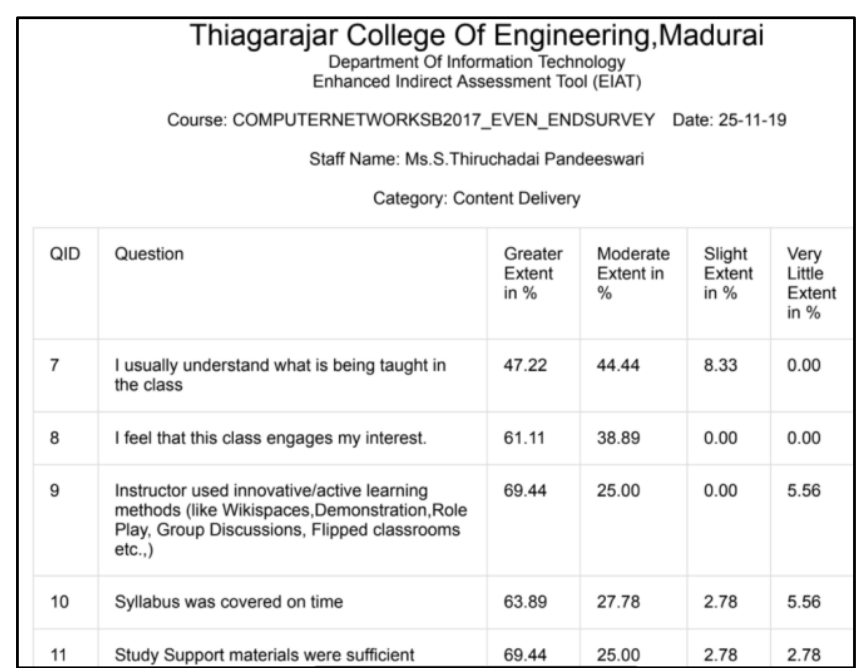

Figure 18- Course Exit Survey - Analysis of parameters about Content Delivery

Qualitative feedbacks were also collected in the Canvas Learning Management System on the quality of lectures, simulations, programming assignments and other assessment items. A snapshot of the qualitative feedback has been presented in figure 19. The positive feedbacks demonstrates that the various instructional design methodologies have motivated the students to learn better .

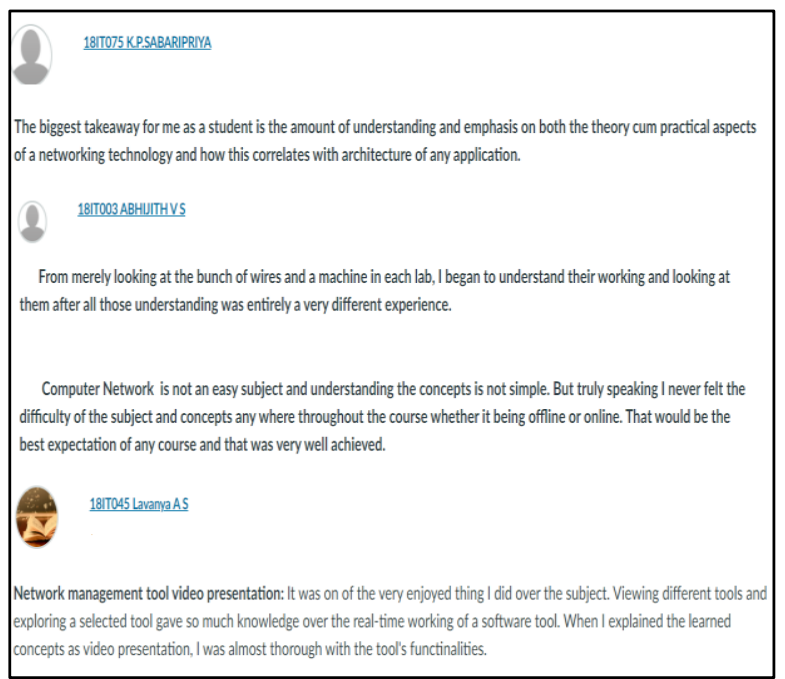

Figure 19 -Qualitative Feedback in Canvas

As a part of content beyond curriculum, learners were encouraged to do certification courses in NPTEL and Open University. The performance of the learners in completion of weekly assignments in NPTEL course has been presented in figure 20 . Nearly $71 \%$ of the students were able to get scores in the range $70-79$ and $17 \%$ of the students were able to score above 80 . These good scores not only demonstrate the engagement of learners but also illustrate the capability of the learners to solve problems at Higher Order Thinking Skills. Around 5\% of the students have successfully completed the certification in the course on "Discovering computer networks: hands on in the Open Networking Lab" in Open University.
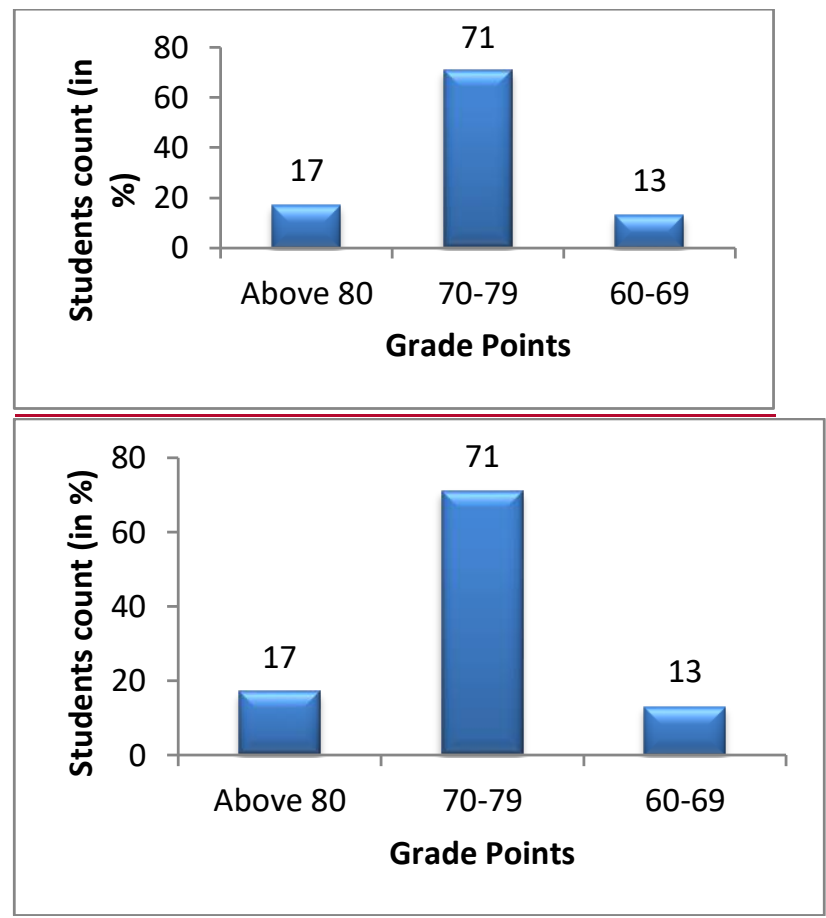

Figure 20-Performance of Learners in Weekly assignments of NPTEL course 


\section{Conclusion}

This research work is carried out with an objective of analysing the impact of a customized instructional design with appropriate active and collaborative learning strategies. Suitable animations, simulations, programming exercises and packet analysis experiments have been selectively included to promote hands on experience and practical skills. The performance of the learners in Continuous assessment test and terminal examinations have significantly improved and is higher than the students admitted in the previous batches. Performance of the students in online courses like NPTEL and open university demonstrates that the leaners are motivated to take up the learning of the course to next level. The positive qualitative feedbacks have motivated the instructors to extend different active and collaborative learning strategies in other related courses.

The research work can be extended in creating a customized instructional design in accordance with different learning styles. Specific approaches can be developed for supporting fast learners and slow learners. The instructional design can also be refined for execution in online and in blended mode.

\section{References}

[1] Knežević, D. Bjelobrk, Vedran Tadić, and Željko Širanović. (2019) Flipped Classroom Model for Advanced Networking Courses.2019 42 ${ }^{\text {nd }}$ International Convention on Information and Communication Technology, Electronics and Microelectronics (MIPRO). IEEE, 2019.

[2] Gao, Yayu, (2018) Improving a Software/Hardware Integrated Computer Networking Laboratory Course." IEEE International Conference on Teaching, Assessment, and Learning for Engineering (TALE). IEEE, 2018.

[3] Guan, Chen-zhi. (2017) Design of computer network experimental course in flipped laboratory mode. $12^{\text {th }}$ International Conference on Computer Science and Education (ICCSE). IEEE, 2017.

[4] Gurgel, P.H., Branco, L.H., Barbosa, E.F. and Branco, K.R., (2013). Development of a practical computer network course through Netkit virtualization tool. Procedia Computer Science, 18, 2583-2586.

[5] Yang, S.K. and Liu, C.G., (2019), r. Promoting the efficiency of learning Computer network course with the use of Cisco network devices, IEEE International Conference on Architecture, Construction, Environment and Hydraulics (ICACEH), 82-85

[6] Zhang, Y., Liang, R. and Ma, H., (2012). Teaching innovation in computer network course for undergraduate students with packet tracer. IERI Procedia, 2, 504-510.

[7] Zhang, L.H. and Li, F., (2016), Application of the spoc teaching mode in courses of computer network in the post-mooc period.,8th International
Conference on Information Technology in Medicine and Education (ITME) 436-440. 